\title{
The Role of a Pre-Fine Needle Aspiration Clinic in Improving the Quality of Thyroid Nodule Investigation in Saskatchewan
}

\author{
Paige Baldwin'1, Terra Arnason², Niomi Singh ${ }^{3}$, Robert Otani ${ }^{4}$, Gary Groot ${ }^{3}$ \\ ${ }^{1}$ College of Medicine, University of Saskatchewan, Saskatoon, Canada \\ ${ }^{2}$ Department of Medicine, Division of Endocrinology, University of Saskatchewan, Saskatoon, Canada \\ ${ }^{3}$ Department of Surgery, University of Saskatchewan, Saskatoon, Canada \\ ${ }^{4}$ Department of Radiology, University of Saskatchewan, Saskatoon, Canada \\ Email: gary.groot@usask.ca
}

How to cite this paper: Baldwin, P., Arnason, T., Singh, N., Otani, R. and Groot, G. (2020) The Role of a Pre-Fine Needle Aspiration Clinic in Improving the Quality of Thyroid Nodule Investigation in Saskatchewan. Open Journal of Radiology, 10, 23-34.

https://doi.org/10.4236/ojrad.2020.101004

Received: January 31, 2020

Accepted: February 28, 2020

Published: March 2, 2020

Copyright $\odot 2020$ by author(s) and Scientific Research Publishing Inc. This work is licensed under the Creative Commons Attribution International License (CC BY 4.0).

http://creativecommons.org/licenses/by/4.0/

\section{(c) (i) Open Access}

\begin{abstract}
Background: The Canadian province of Saskatchewan introduced a pre-fine needle aspiration (FNA) clinic to review adherence of referrals for thyroid biopsy based on the guidelines of the American College of Radiology's (ACR) Thyroid Imaging, Reporting and Data System (TI-RADS) scoring system. The intention is to minimize low-yield biopsy rates by improving the quality of thyroid nodule investigation in Saskatchewan through this clinic. TI-RADS is a malignancy risk scoring system for thyroid nodules based on five sonographic characteristics: composition, echogenicity, shape, margin, and echogenic foci (calcium). Recommendations for intervention or clinical follow-up are further determined by the size of the nodule. Methods: Through a retrospective chart review of all thyroid biopsy referrals to the Royal University Hospital (RUH) in Saskatchewan between 22 March 2016 and 17 May 2018, the impact of the multidisciplinary pre-FNA clinic on appropriate thyroid biopsies in Saskatchewan was evaluated. Results: This study evaluated 252 referrals, 203 of which underwent FNA and 23 which received surgical biopsy. TI-RADS scores appended to thyroid biopsy referrals increased upon pre-FNA clinic initiation, yet score quality did not improve. Rates of malignant biopsies were lower than ACR-reporting suggesting inappropriate biopsy of low risk nodules perhaps by overcalling the TI-RADS score. The majority of FNA cytology matched final surgical pathology, with $78 \%$ of indeterminate FNAs being malignant, and all non-diagnostic FNAs being benign. Conclusions: The implementation of the pre-FNA clinic reduced the number of thyroid biopsies in Saskatchewan by $11 \%$ overall.
\end{abstract}




\section{Keywords}

Thyroid Nodules, Thyroid Cancer, Fine Needle Aspiration Biopsy, Thyroid Imaging Reporting and Data System (TIRADS)

\section{Introduction}

Thyroid nodules are frequently found in the adult population, many incidentally by imaging being done for other reasons. In a randomly-selected group, thyroid nodules can be detected in up to $68 \%$ of individuals by high-resolution ultrasound (US) [1] [2]. While the majority of these cases are generally benign, some of these cases can be malignant [2] [3]. The malignant cases are important to identify and treat, yet the number of unnecessary benign biopsies should be minimized. To address this issue, the American College of Radiology (ACR) introduced the ultrasound-based Thyroid Imaging, Reporting and Data System (TI-RADS) in 2015 with the publication of their lexicon [4]. TI-RADS was designed based on the BI-RADS ${ }^{\oplus}$ risk classification commonly used in breast imaging [5] [6].

There are five levels within TI-RADS, from TR1 (benign) to TR5 (high suspicion of malignancy) [7]. These levels are based on five sonographic feature categories. When assessing the ultrasound of a thyroid nodule, points are awarded based on the presence or absence of particular findings within each category. One feature with the highest risk associated with it is chosen for each of the following categories: composition, echogenicity, shape, and margin, while all the features that apply in the final category of echogenic foci are included for summative points [7]. Each feature is awarded a pre-determined number of points. The sum of these points determines the TI-RADS level (TR) of the nodule [7]. Recommendations for the type of treatment at each level are further determined by the size of the nodule. TR1 (benign) and TR2 (not suspicious) are not recommended for fine-needle aspiration (FNA). TR3 (mildly suspicious), TR4 (moderately suspicious), and TR5 (highly suspicious) are recommended for FNA if they measure over $2.5 \mathrm{~cm}, 1.5 \mathrm{~cm}$, and $1.0 \mathrm{~cm}$, respectively. The same three levels are recommended for follow-up clinically and/or with US if they are below the previously listed size but above $1.5 \mathrm{~cm}, 1.0 \mathrm{~cm}$, and $0.5 \mathrm{~cm}$, respectively.

Implementation of TI-RADS was discussed at the provincial multidisciplinary thyroid cancer rounds for Saskatchewan in early 2016. A multidisciplinary pre-FNA clinic consisting of local thyroid experts in radiology, surgical oncology, otolaryngology, nuclear medicine, and endocrinology was created. The intention was to improve the quality of the investigation of thyroid nodules in Saskatchewan and to minimize the low-yield biopsy rate. As of late March 2016, all referrals for US-guided thyroid FNA biopsies sent to the Royal University Hospital (RUH) were filtered through the pre-FNA clinic. The clinic members 
met twice a month to review the US imaging and relevant reports and to assign independent TI-RADS scores. Feedback was then sent to the referring physicians from the pre-FNA clinic advising on the appropriateness of a biopsy, including information on the role of TI-RADS in their decisions.

Increasing the efficacy and efficiency of the Saskatchewan health system is important to provide the best possible standard of care to patients. We hypothesized that implementation of the pre-FNA clinic will lead to a reduction in unnecessary thyroid biopsies being completed and increase TI-RADS use and reporting by local radiologists. We performed an audit on follow-up management of non-diagnostic and indeterminate cytology in Saskatchewan, as well as the prevalence of malignant cytology within each TI-RADS level and compared these results with American Thyroid Association (ATA) and ACR guidelines, respectively. Finally, we compared the accuracy of local FNA cytology results with final surgical pathology.

\section{Methods}

A retrospective chart review was completed for all thyroid biopsy referrals to the RUH in Saskatchewan. All new thyroid biopsy referrals sent to the RUH between 22 March 2016 and 17 May 2018 that were screened by the multidisciplinary pre-FNA clinic were included. De-identified data was collected from referral letters, pathology reports, pre-FNA clinic charts, and imaging reports.

A total of 286 referrals were collected (Figure 1). Five referrals were excluded based on missing information: one because the pre-FNA clinic failed to assign and/or record a TI-RADS score; two lacked an accurate electronic health number; one was a parathyroid biopsy referral; and one was a duplicate referral. Referrals related to 28 multinodular patients were limited by the caveat that only certain nodules were biopsied whereas others were not, therefore, 29 referrals not biopsied that met these criteria were excluded. Pre-biopsy analysis was performed on this data including a total of 252 referrals.

Data was then separated based on whether or not a biopsy and/or surgery was performed. Further analysis on pathology reports was performed for 203 referrals from FNA and 23 patients from surgery. FNA cytology results were compared to ACR-predicted cancer rates depending on TI-RADS score [7]. The follow-up management steps for indeterminate and non-diagnostic FNA results were compared with best practices based on ATA guideline recommendations [8].

FNA cytology reporting was based on the Bethesda system's six categories: non-diagnostic, benign, atypia of unknown significance (AUS)/follicular lesion of unknown significance (FLUS), (suspicious for a) follicular neoplasm (FN), suspicious for malignancy, and malignancy [9]. For the purposes of this study we reduced these categories down to four groups: non-diagnostic, benign, indeterminate (AUS/FLUS and FN), and malignant (suspicious for malignancy and malignancy). 


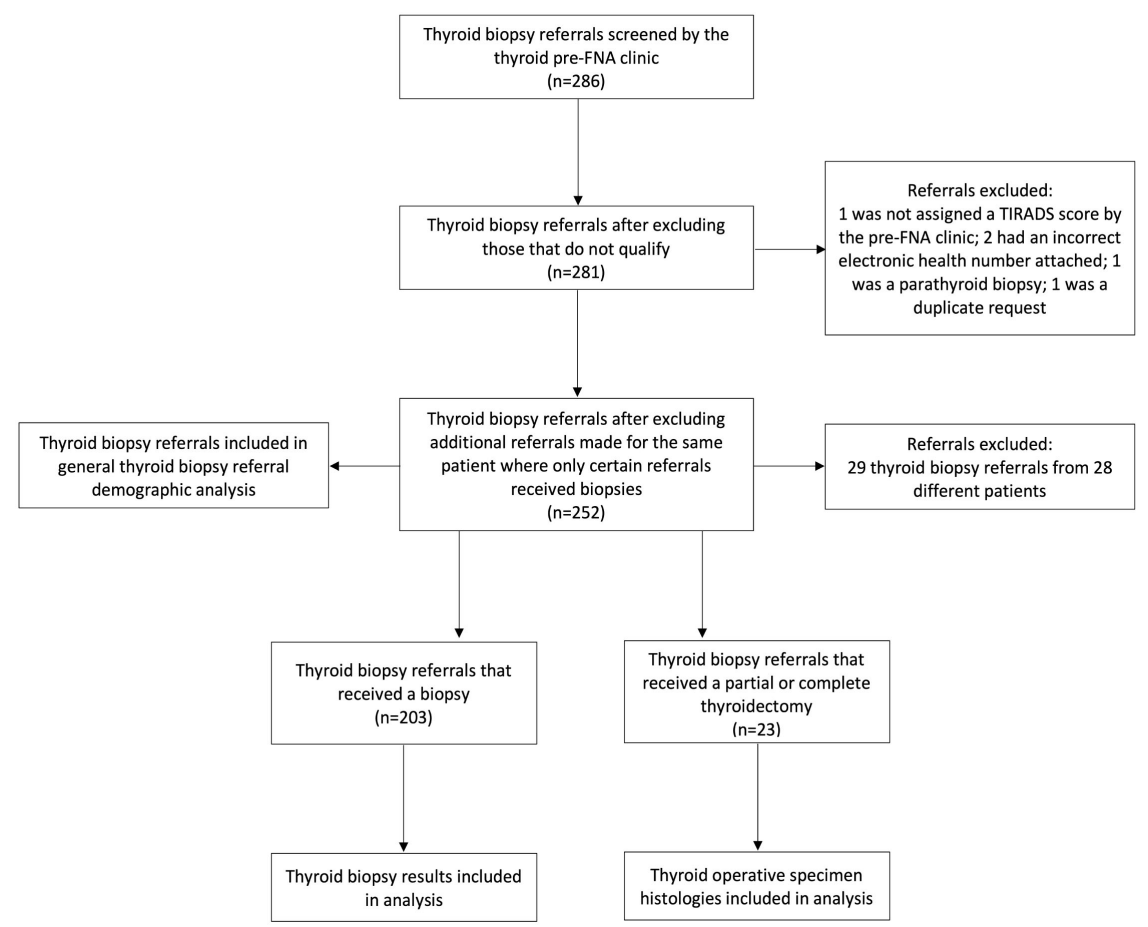

Figure 1. A flow chart demonstrating the selection process for appropriate data for each stage of analysis in this study.

The raw data from this study is reported in complete sets without statistical manipulation. The data was converted to percentages for ease of comparison to the guidelines provided by the ATA [8], in order to audit local practice to that recommended by the ATA, as well as compliance over time.

\section{Results}

\subsection{Study Population}

Of the 252 referrals received between 22 March 2016 and 17 May 2018 by the pre-FNA clinic, 190 referrals (75\%) arrived without a TI-RADS score, while 62 referrals (25\%) had a previously assigned TI-RADS score. The percentage of referrals arriving with a score increased over time from $0 \%$ in 2016 to $38 \%$ in the first half of 2018 (Figure 2). The percentage of pre-scored referrals that had their scores altered by the pre-FNA clinic was $100 \%$ (1) in 2016, 45\% (5) in early 2017 , $42 \%$ (11) in late 2017, and 54\% (13) in early 2018.

\subsection{Pre-Screening Recommendations for Biopsy}

After TI-RADS score adjustments by the pre-FNA clinic, there was one referral for a TR1 nodule, ten referrals for TR2 nodules, 50 referrals for TR3 nodules, 87 referrals for TR4 nodules, and 104 referrals for TR5 nodules. The pre-FNA clinic recommended 225 of the 252 referrals (89\%) continue on to a biopsy (Figure 3). None of the TR1 or TR2 nodules were recommended for FNA by the pre-FNA clinic, and an explanatory letter was sent to the referring physician. Of the nodules determined to be TR3, $80 \%$ (43) were recommended for FNA by the 


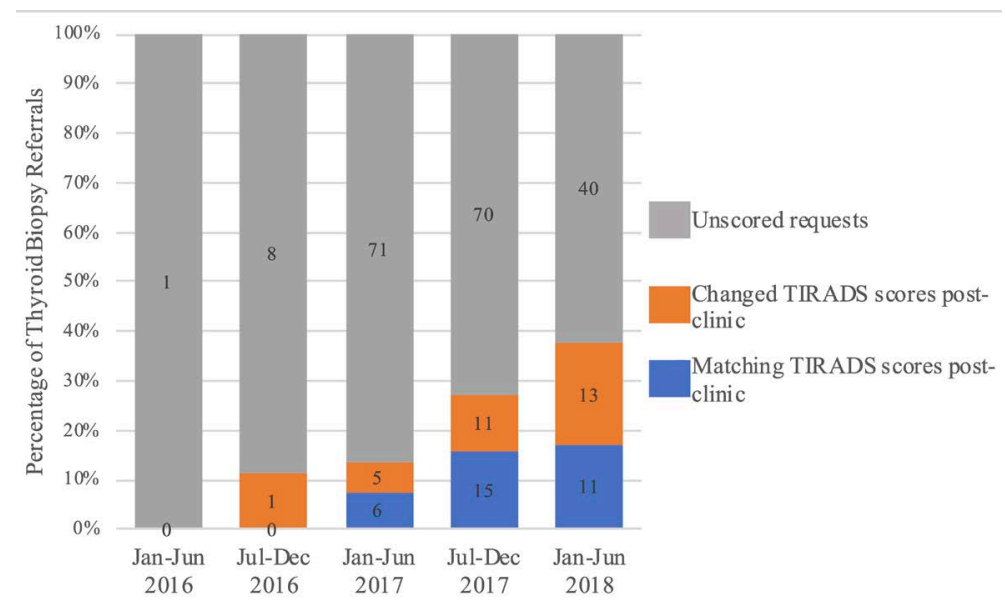

(a)

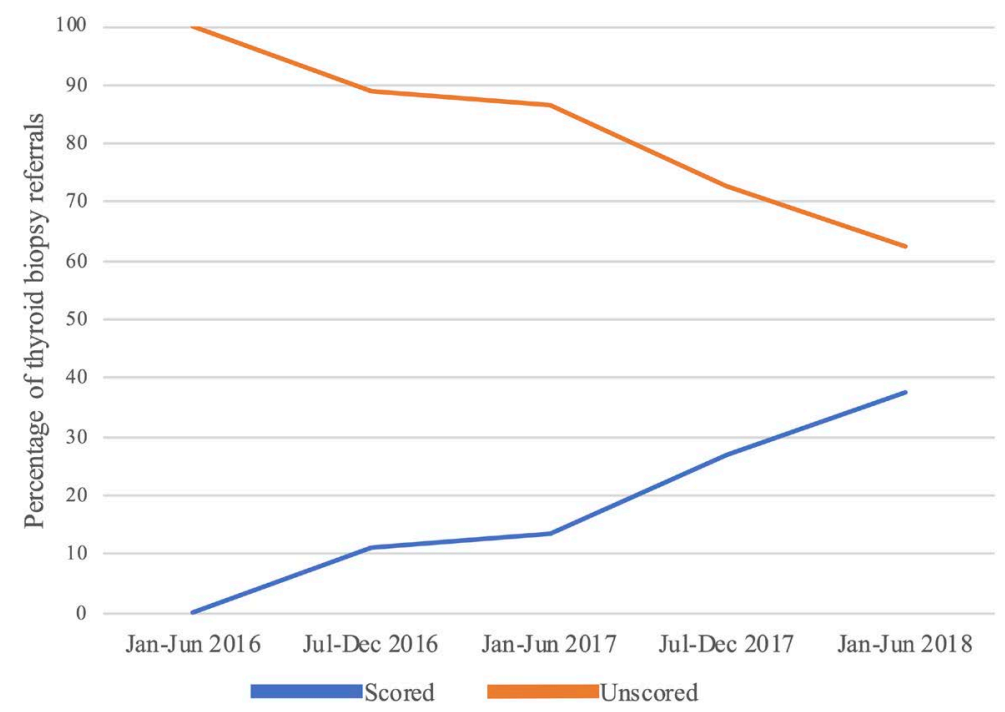

(b)

Figure 2. Changes over time in the inclusion of TI-RADS scores with thyroid biopsy referrals and how they compare to those given by the pre-FNA clinic.

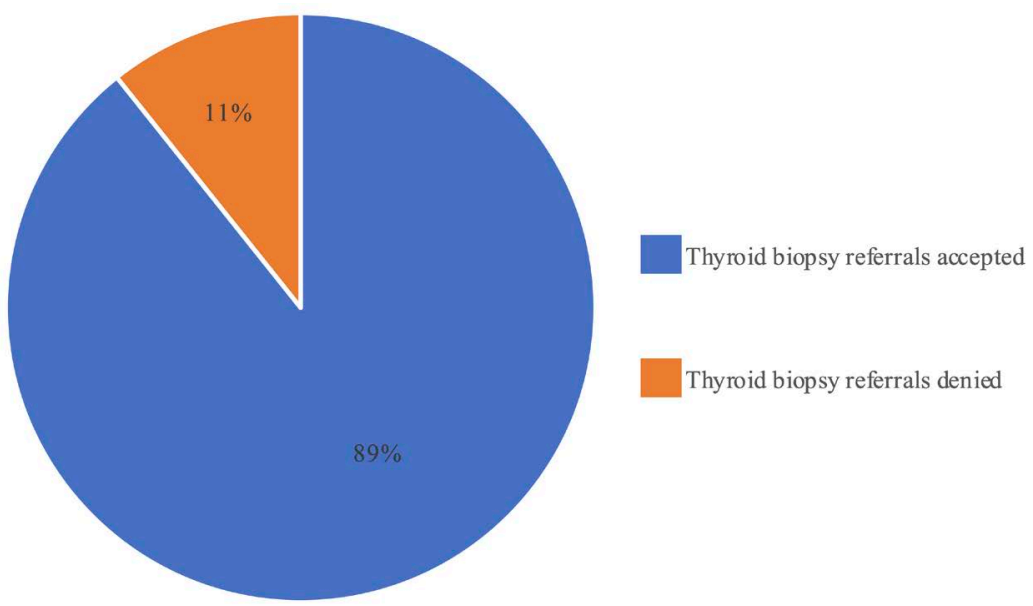

Figure 3. Pie chart demonstrating the effect of the pre-FNA clinic on reducing thyroid biopsies in Saskatchewan by promoting or denying referrals. 
pre-FNA clinic. Recommendation for biopsy was made on $94 \%$ (82) of the TR4 nodules. The pre-FNA clinic recommended $96 \%$ (100) of the TR5 nodules continue on to biopsy. The TR3-TR5 nodules not recommended for biopsy were too small and were instead recommended for clinical follow-up.

\subsection{Results of Biopsy Pathology Correlated to Adjusted TI-RADS Score}

Two TR2 nodules were biopsied and cytology results for both were benign. Of the 41 TR3 nodules biopsied, 80\% (33 nodules) were benign, 5\% (2 nodules) were non-diagnostic, 12\% (5 nodules) were indeterminate, and 2\% (1 nodule) were malignant. Cytology results for the 70 TR4 nodules biopsied, showed 70\% (49) were benign, $7 \%$ (5) were non-diagnostic, $21 \%$ (15) were indeterminate, and 1\% (1) was malignant. There were 90 TR5 nodules biopsied, with cytology results as follows: $67 \%$ (80) benign, $9 \%(8)$ non-diagnostic, $14 \%$ (13) indeterminate, and 10\% (9) malignant (Figure 4).

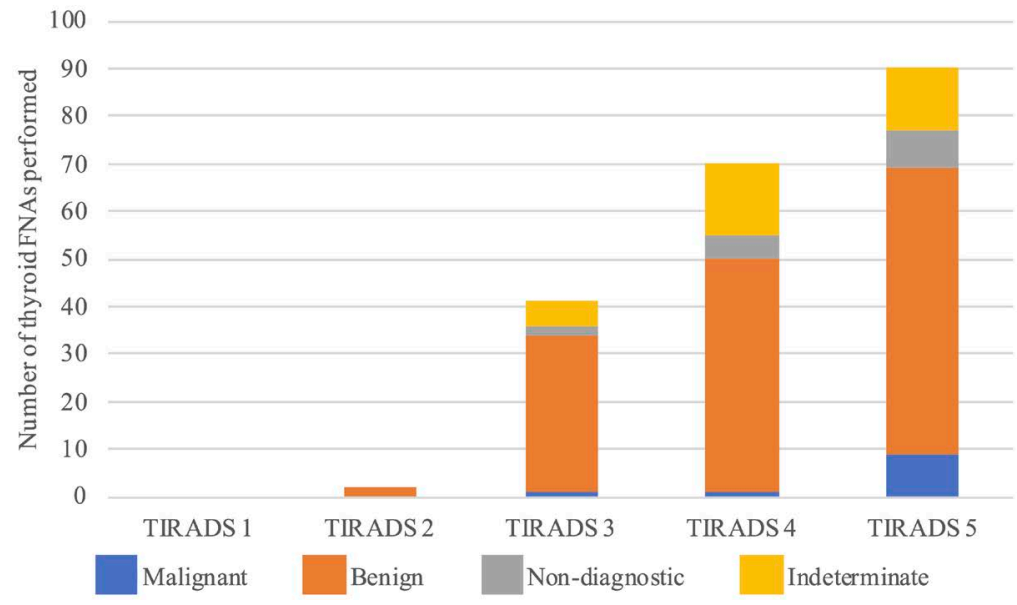

(a)

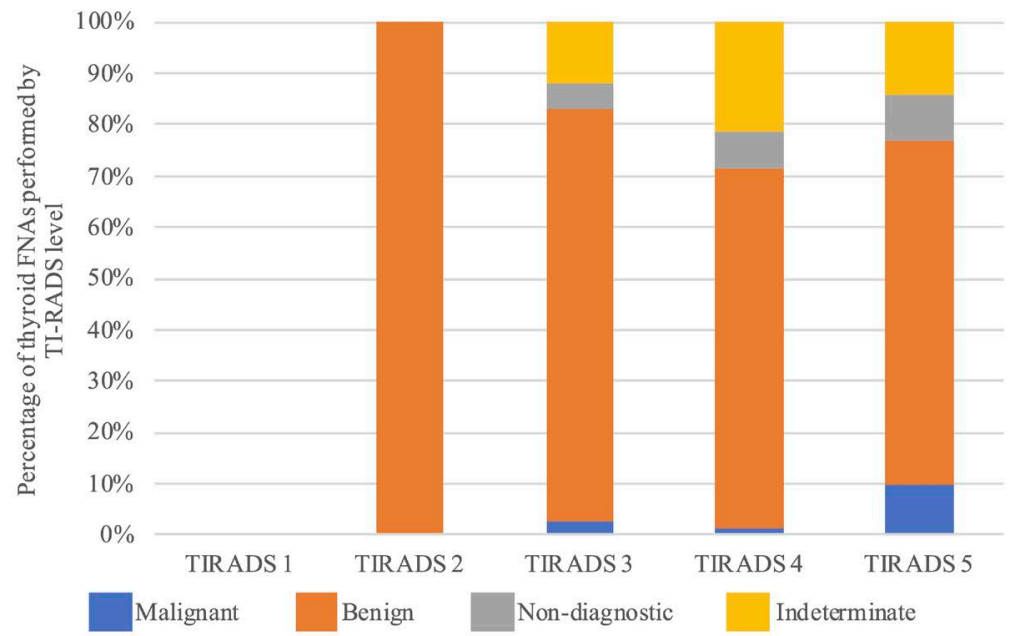

(b)

Figure 4. Thyroid FNA cytology results sorted by TI-RADS level based on number of FNAs (a) and percentage of FNAs (b). 
The biopsies with indeterminate cytology received various types of follow-up (Figure 5). Surgery was ultimately performed on $34 \%$ (10) of these patients. Another 13\% (4) underwent a repeat FNA. For 3\% (1) of these patients, nuclear medicine imaging was pursued to determine if the nodules were functioning. Ongoing clinical follow-up was being pursued by $50 \%$ (15) of these patients at the time of this study.

The biopsies which received non-diagnostic cytology results underwent similar types of follow up to that of the indeterminate nodules (Figure 6). Surgery was ultimately performed on $15 \%$ (2) of these patients, and US on $8 \%$ (1). Another 8\% (1) of these patients had repeat FNA, while 69\% (9) of them were still pursuing ongoing clinical follow up at the time of this study.

\subsection{Results of Surgery}

From the 203 patients who received an FNA, 10\% (21) went on to have surgery (Figure 7). Four of these patients had a benign FNA cytology and all four had a

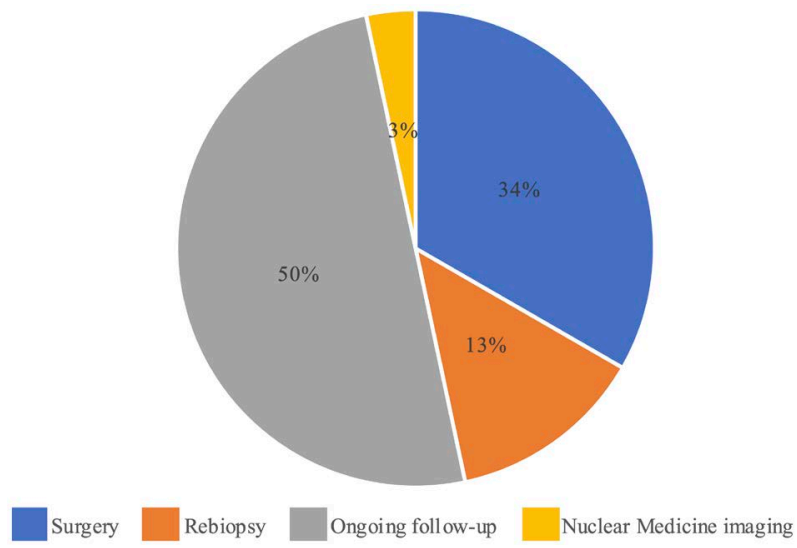

Figure 5. Pie chart demonstrating the methods of follow-up care for patients with indeterminate cytology results in percentages.

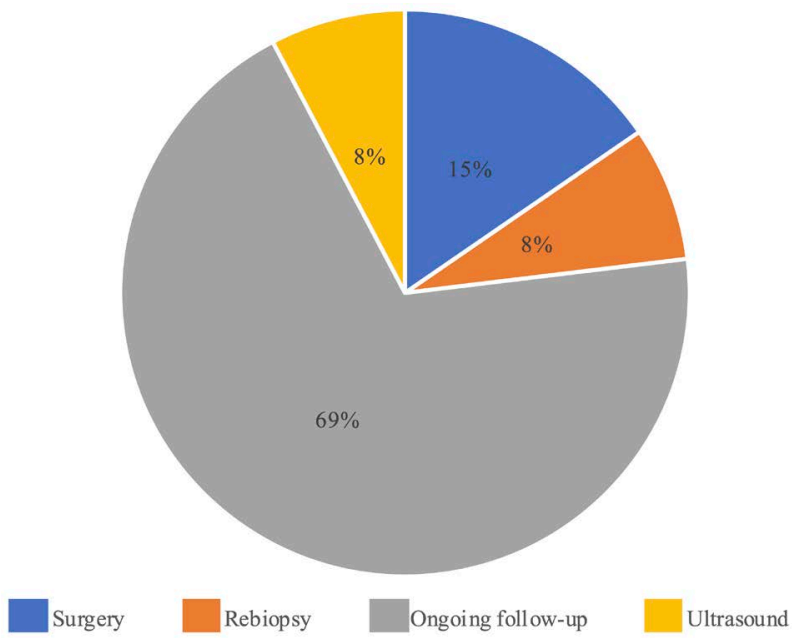

Figure 6. Pie chart demonstrating the methods of follow-up care for patients with non-diagnostic cytology results in percentages. 


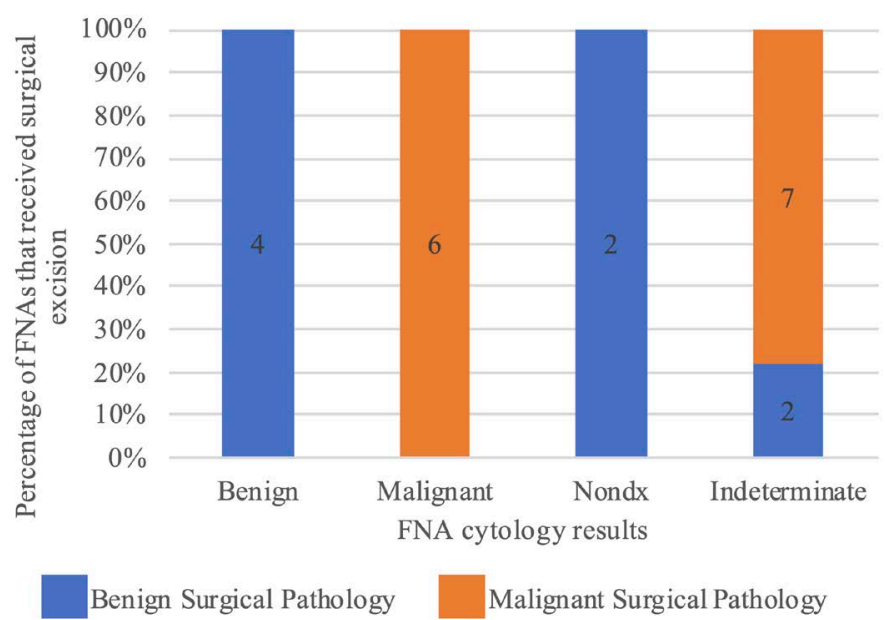

Figure 7. Surgical pathology sorted by FNA cytology results for patients that received surgical excision following FNA.

benign final pathology. Of the 6 patients who had a malignant FNA cytology and went to surgery, all had a malignant final pathology. Two individuals with a non-diagnostic FNA went to surgery and both had benign final surgical pathology results. Of the 9 patients who received an indeterminate biopsy result and went to surgery, 2 had a benign final surgical pathology and 7 had a malignant final surgical pathology.

\section{Discussion}

Use of formal TI-RADS risk stratification by radiologists in Saskatchewan for malignancy of thyroid nodules based on ultrasound findings has been increasing since the implementation of the pre-FNA clinic (Figure 2). This change is following best practice suggestions by international thyroid cancer expert groups, including the ATA [8] [9] [10]. Inclusion of a TI-RADS score with referrals for thyroid biopsies has almost quadrupled from 2016 to 2018. Despite the increase in TI-RADS use, accuracy of TI-RADS scores provided by community radiologists compared with the pre-FNA clinic has not been increasing. This highlights the fact that there may be an aspect of user expertise in reporting. In fact, more scores had to be changed in 2018 than in 2017. This discovery acknowledges the possible role of the pre-FNA clinic in improving awareness and implementation of TI-RADS. It cannot be determined by this study alone whether the increase in use of TI-RADS locally is a by-product of the feedback provided by the pre-FNA clinic to referring physicians or simply due to more radiologists discovering TI-RADS on their own. However, the pre-FNA clinic has been important in reducing the number of thyroid biopsies by $11 \%$ since implementation through re-evaluation of thyroid sonography for each referral (Figure 3). Inaccuracy of pre-assigned TI-RADS scores compared with those given by the pre-FNA clinic suggests a possible need for additional education for local radiologists on the utilization of TI-RADS. An easy method of improving accuracy might be to raise awareness of scoring tools that aid the radiologists and technicians in including 
and identifying relevant sonographic features [10] [11]. One such tool was created locally and is used by the pre-FNA clinic when scoring each referral [11].

When reviewing the FNA cytology, it can be noted that benign biopsies were prevalent ( $71 \%$ of FNAs performed). Both of the TR2 patients who received a biopsy, despite recommendation against it by the pre-FNA clinic, had benign results, supporting the clinic's decision and ACR guidelines [7]. ACR guidelines discussing TI-RADS scoring describe a predictive malignancy rate of $5 \%$ for TR3 nodules, $5 \%$ - 20\% for TR4 nodules, and more than $20 \%$ for TR5 nodules [7]. With a $2 \%$ malignancy rate for TR3, $1 \%$ for TR4, and $10 \%$ for TR5, Saskatchewan is experiencing much lower ratios of malignant to benign biopsies than ACR guidelines suggest (Figure 4).

There are several possible reasons to explain our low malignant biopsy rates. First, unfamiliarity with TI-RADS due to its recent local implementation may be causing physicians to err on the side of caution by assigning higher scores to prevent missing a malignancy. Second, Saskatchewan thyroid malignancy rates and presentations could differ from American rates and presentations. Finally, our Provincial high rates of the "indeterminate" Bethesda pathology category by the pathologists reviewing FNA biopsies may be reducing our malignancy rates. ATA guidelines recommend this category be used sparingly, with only around $7 \%$ of biopsies bearing this title, however indeterminate results represent $16 \%$ of our local thyroid biopsies. In reality the ATA is seeing rates of $1 \%-27 \%$ for indeterminate biopsy results [8]. While we are still within that range, we are at the higher end, and more than double the recommended $7 \%$.

Solutions to this problem may lie with more education and practice for radiologists and technicians to increase comfort in assigning lower scores. Further studies in other locations or with larger local sample sizes may confirm local differences in thyroid malignancy rates and presentations compared to ATA guidelines. More education for pathologists performing the FNA cytology focused on the use of the indeterminate classifications could also increase malignancy rates by reducing indeterminate ones.

ATA guidelines have recommendations for the management of indeterminate or non-diagnostic FNA results [8]. Indeterminate results, AUS and FLUS, are weakly recommended for a repeat FNA and strongly recommended for surgical excision or surveillance. For the indeterminate result, surgical excision is the standard of care. Half of our indeterminate biopsies received some sort of active investigation: $34 \%$ were sent for surgical excision, $13 \%$ received a repeat FNA, both in accordance with ATA guidelines, and 3\% underwent nuclear medicine imaging as an alternative version of surveillance (Figure 5). The other $50 \%$ of indeterminate biopsies were still pursuing ongoing clinical care at the time of this study. Non-diagnostic results are strongly recommended for a repeat biopsy under US guidance [8]. A weak recommendation is given for non-diagnostic results to have surgical excision if sonographic features are highly suspicious for malignancy, or to continue surveillance. A repeat biopsy was performed on one of our 13 non-diagnostic FNA patients (8\%), in accordance with ATA guidelines 
(Figure 6). Surgical excision was pursued by 2 of these patients (15\%), both with TR5 nodules, which corresponds with the ATA recommendation for high-risk nodules [8]. One patient (8\%) received an US as follow-up. For both the indeterminate and non-diagnostic FNA patients, there was overlap of the cut-off and data collection which provides a limitation in making conclusions about follow-up care. The majority of these cases, 9 of our 13 (69\%) non-diagnostic and 15 of our 30 indeterminate (50\%), were still pursuing ongoing clinical follow-up at the time of the study. While some of these patients may not have received follow-up care yet, others might have opted for active surveillance, which was not part of the data collected, but which would comply with ATA guidelines [8].

Of those patients who received an FNA and went on to receive surgical excision, we were most interested in those with a surgical pathology that did not match the pre-operative FNA pathology report. By comparing pathology results, we hoped to determine the reliability of our local FNA cytology. All nodules with a benign or malignant FNA cytology had a matching surgical excision pathology (Figure 7). Of the indeterminate surgical excisions, 78\% were malignant, which suggests that surgical excision for indeterminate biopsies is appropriate locally, as is recommended by ATA guidelines, and perhaps we should be referring more of our indeterminate biopsies in Saskatchewan for surgical excision. On the other hand, while we only sent two non-diagnostic FNA patients for surgical excision, both were benign, supporting ATA guidelines to repeat the FNA rather than excise the nodule [8].

One of the limitations of our study was the narrow scope of referrals that were included, as we only covered the referrals received by the RUH and not the entire metro area or our second major urban centre, Regina, Saskatchewan. This reduced our sample size and limited our ability to make generalizations about the entire province. Another limitation we faced, as previously mentioned, was that a large portion of our sample size was still receiving ongoing clinical care at the time of this study and therefore inclusion of final surgical pathology in comparison to FNA pathology was not possible (Figure 5 and Figure 6). Due to the longitudinal nature of this clinical care, with recommendations for follow-up spanning 12 months or more, the final outcomes of these cases were unknown at the time of this study. These unknown outcomes further limited our ability to draw stronger conclusions from the data.

\section{Conclusion}

The implementation of the pre-FNA clinic reduced the number of thyroid biopsies by $11 \%$ presumably by limiting the procedures performed to those with optimal indications. Inclusion of TI-RADS scores with thyroid biopsy referrals has increased since the implementation of the pre-FNA clinic in Saskatchewan and appears to be very useful when reported accurately to stratify the appropriate indication for thyroid nodule biopsy. Local rates of malignant thyroid biopsies were lower than ACR guidelines due to various possible factors. The majority of FNA cytology matched final surgical pathology. 


\section{Acknowledgements}

The authors confirm there was no significant funding for this project that would have influenced outcomes. We would like to thank the University of Saskatchewan, College of Medicine for funding this project.

\section{Conflicts of Interest}

The authors declare no conflicts of interest regarding the publication of this paper.

\section{References}

[1] Guth, S., Theune, U., Aberle, J., Galach, A. and Bamberger, C.M. (2009) Very High Prevalence of Thyroid Nodules Detected by High Frequency (13 MHz) Ultrasound Examination. European Journal of Clinical Investigation, 8, 699-706. https://doi.org/10.1111/j.1365-2362.2009.02162.x

[2] Dean, D.S. and Gharib, H. (2008) Epidemiology of Thyroid Nodules. Best Practice \& Research Clinical Endocrinology \& Metabolism, 6, 901-911. https://doi.org/10.1016/j.beem.2008.09.019

[3] Papini, E., Guglielmi, R., Bianchini, A., Crescenzi, A., Taccogna, S., Nardi, F., Panunzi, C., Rinaldi, R., Toscano, V. and Pacella, C.M. (2002) Risk of Malignancy in Nonpalpable Thyroid Nodules: Predictive Value of Ultrasound and Color-Doppler Features. Journal of Clinical Endocrinology and Metabolism, 5, 1941-1946. https://doi.org/10.1210/jcem.87.5.8504

[4] Grant, E.G., Tessler, F.N., Hoang, J.K., Langer, J.E., Beland, M.D., Berland, L.L., Cronan, J.J., Desser, T.S., Frates, M.C., Hamper, U.M. and Middleton, W.D. (2015) Thyroid Ultrasound Reporting Lexicon: White Paper of the ACR Thyroid Imaging, Reporting and Data System (TIRADS) Committee. Journal of the American College of Radiology, 12, 1272-1279. https://doi.org/10.1016/j.jacr.2015.07.011

[5] D’Orsi, C.J., Sickles, E.A., Mendelson, E.B., Morris E.A., et al. (2013) ACR BI-RADS Atlas, Breast Imaging Reporting and Data System. American College of Radiology, Reston, VA.

[6] Mercado, CL. (2014) BI-RADS Update. Radiologic Clinics, 52, 481-487. https://doi.org/10.1016/j.rcl.2014.02.008

[7] Tessler, F.N., Middleton, W.D., Grant, E.G., Hoang, J.K., Berland, L.L., Teefey, S.A., Cronan, J.J., Beland, M.D., Desser, T.S., Frates, M.C. and Hammers, L.W. (2017) ACR Thyroid Imaging, Reporting and Data System (TI-RADS): White Paper of the ACR TI-RADS Committee. Journal of the American College of Radiology, 5, 587-595. https://doi.org/10.1016/j.jacr.2017.01.046

[8] Haugen, B.R., Alexander, E.K., Bible, K.C., Doherty, G.M., Mandel, S.J., Nikiforov, Y.E., Randolph, G.W., Sawka, A.M., Schuff, K.G., Sherman, S.I. and Ann, S. (2016) 2015 American Thyroid Association Management Guidelines for Adult Patients with Thyroid Nodules and Differentiated Thyroid Cancer: The American Thyroid Association Guidelines Task Force on Thyroid Nodules and Differentiated Thyroid Cancer. Thyroid. https://doi.org/10.1089/thy.2015.0020

[9] Cibas, E.S. and Ali, S.Z. (2009) The Bethesda System for Reporting Thyroid Cytopathology. American Journal of Clinical Pathology, 5, 658-665. https://doi.org/10.1309/AJCPPHLWMI3JV4LA

[10] Tessler, F.N., Middleton, W.D., and Grant, E.G. (2018) Thyroid Imaging Reporting 
and Data System (TI-RADS): A User's Guide. Radiology, 1, 29-36.

https://doi.org/10.1148/radiol.2017171240

[11] Wang, J.T., Ellchuk, T., Otani, R., Groot, G. and Babyn, P. (2018) Online TI-RADS Calculator. Open Journal of Radiology, 8, 175-180.

https://doi.org/10.4236/ojrad.2018.83020 